\title{
Failed Back Surgeries and Minnesota Multiphasic Personality Inventory (MMPI) Profiles
}

\author{
Linas A. Bieliauskas, ${ }^{1-3}$ Gregory P. Graziano, ${ }^{1,2}$ Kristen Kullgren, ${ }^{2}$ \\ and Brad L. Roper ${ }^{2}$
}

MMPI profiles were evaluated for 105 prospective surgical patients who had previously undergone surgery or other procedures for treatment of back pain. Patients were classified into groups having undergone zero, one, two, three, or four or more previous surgeries. While all groups demonstrated a characteristic somatogenic profile, none of the MMPI validity or clinical scales significantly differentiated the groups and there was no relationship between increased number of surgeries and MMPI scale characteristics. These results support the nonoptimistic prognostication of the somatogenic MMPI profile for surgical intervention for back pain but show no clear relationship of MMPI profile characteristics to degree of experience of previously failed surgery.

KEY WORDS: low back pain; Minnesota Multiphasic Personality Inventory (MMPI); back surgery; psychological assessment.

\section{INTRODUCTION}

It has been estimated that less than $50 \%$ of patients undergoing back surgery experience complete relief from pain (Chapman \& Bonica, 1985). Given the influence of higher cortical centers on pain experience, as postulated by gate control theory, it might be expected that the degree of pain relief following surgery may interact with personality variables. Sternbach, Wolf, Murphy, and Akeson (1973) reported a characteristic Minnesota Multiphasic Personality Inventory (MMPI; Hathaway \& McKinley, 1983)

${ }^{1}$ V.A. Medical Center, Ann Arbor, Michigan 48105.

${ }^{2}$ University of Michigan, Ann Arbor, Michigan 48109.

${ }^{3}$ To whom correspondence should be addressed at Psychology Service (116B), V.A. Medical Center, 2215 Fuller Road, Ann Arbor, Michigan 48105. 
profile for patients with chronic back pain as distinguished from patients with acute back pain; in comparison, the former had relative elevation on or over a $T$ score of 70 on the Hypochondriasis (Hs), Depression (D), and Conversion Hysteria (Hs) scales. Bradley, Prokop, Margolis, and Gentry (1978) identified subgroups of patients with low back pain via cluster analysis of MMPI profiles. They reported one subgroup of female patients and one subgroup of male patients with sole elevations above a $T$ score of 70 on scales $\mathrm{Hs}, \mathrm{D}$, and Hy. Bradley et al. note that these patient subgroups resemble a similar profile type identified by Sternbach (1974) which might be classified as somatogenic and that such patients likely are poor candidates for intervention. There have been multiple similar reports. Pheasant, Gilbert, Goldfarb, and Herron (1979) found elevations on scales Hs and Hy to be inversely related to surgical outcome and that the somatogeniclike profile "was associated with poor surgical outcome, especially for multiply-operated cases" (p. 83). Wifling, Klonoff, and Kokan (1973) found the somatogenic profile to be a characteristic of patients undergoing multiple surgeries. Wiltse and Rocchio (1975) reported that elevations on Hs and Hy scales, in combination with physician ratings of likelihood of functional etiology, are predictive of poor outcome for patients undergoing chymopapain injection, while Blumetti and Modesti (1976) reported cutoff scores of the $\mathrm{Hs}$ and $\mathrm{Hy}$ scales in the clinically significant range as being predictive of poor surgical outcome for back pain. Maruta, Swanson, and Swenson $(1976 \mathrm{a}, \mathrm{b})$ also report similar profiles being associated with lowback pain patients who are resistant to medical and surgical intervention. The value of elevations on the Hs and Hy scales have been confirmed more recently by Strassberg, Reimherr, Ward, Russell, and Cole (1981) in predicting response to medical or psychological treatment for various kinds of chronic pain, and Strassberg, Tilley, Bristone, and Oei (1992) describe the somatogenic-like profile as similarly characteristic of Australian chronic pain patients as well as American chronic pain patients.

We had the opportunity to study MMPI personality measures in a group of patients who had varying numbers of previously failed back surgeries or other procedures and who were being evaluated for further surgery. Since most of these patients had previously undergone back surgery without success, it was expected that they may evidence the somatogenic profile on the MMPI as has been previously reported. However, since these patients represented a range of previous numbers of surgeries, we proposed to investigate the potential relationship between MMPI scale elevations and configurations and increasing numbers of failed surgical procedures. It was predicted that increased numbers of failed surgeries would be reflected in either increased scores on scales related to psychological distress or significantly different personality characteristics. 


\section{METHOD}

Subjects were 105 outpatients (45 males and 60 females) being evaluated for surgery to relieve back pain. Patients were selected based upon a history of failed previous lumbar surgery, a history of compensation litigation, and/or previous psychiatric history (Spengler \& Greeman, 1979). All patients had complaints of mechanical low-back pain as a continuing reason for their low-back disability and all had an objective anatomic abnormality as identified on either MRI or CT myelographic imaging studies. All patients received an MMPI to complete and return as part of their evaluation. All patients also were administered the Shipley Institute of Living Scale (Zachary, 1991) to obtain an estimated IQ and ensure that patients adequately understood the MMPI items. Patients were also asked to complete a demographic questionnaire which included an indication of the number of previous back operations they had undergone.

MMPI $T$ scores were calculated for each patient and patients were assigned to groups according to the number of previous back surgeries with the following resulting numbers:

$$
\begin{aligned}
& 0 \text { previous surgeries: } 14 \\
& 1 \text { previous surgery: } 43 \\
& 2 \text { previous surgeries: } 27 \\
& 3 \text { previous surgeries: } 11 \\
& 4+\text { previous surgeries: } 10
\end{aligned}
$$

Multivariate, as well as one-way analysis of variance, was conducted on the MMPI scales to detect differences between these groups.

\section{RESULTS}

All patients scored an estimated IQ of 85 or greater on the Shipley Institute of Living Scale. None of the individual MMPI validity or clinical scales differed significantly between the groups $[F(5,98)$ between .279 and 1.470 for all scales, all $p$ 's $>.05]$. All groups showed elevations between $T$ scores of 70 and 80 on scales Hs and Hy, between $T$ scores of 65 and 75 on scale $D$, and between $T$ scores of 60 and 69 on scale PD, the most commonly described MMPI scales in groups of patients with chronic pain. Multivariate analysis of variance similarly did not show significant differences between numbers of surgeries on the MMPI combined clinical scales $[F(5,40)=1.004, p=.468]$. There was no trend on any of the scales for 
any direct relationship between number of prior surgeries and the clinical scales of the MMPI. (See Fig. 1.)

\section{DISCUSSION}

All groups of patients demonstrated the somatogenic MMPI profile of elevated scores on scales $\mathrm{Hs}, \mathrm{D}$, and $\mathrm{Hy}$, although scale $\mathrm{D}$ was a few $T$ score points below 70 for several of the groups. Thus, the expected profile emerged in groups of patients who either had previously failed to benefit from other medical procedures or surgical intervention for back pain or were suspected of having psychiatric difficulties which might indicate a lowered probability of successful surgical intervention. However, there was no support for a relationship between number of prior failed surgeries for back pain and increased levels of psychological distress. There was also no suggestion of any unique relationship between personality characteristics (as measured by the MMPI) and numbers of failed back surgery procedures. In fact, the profiles generated by all groups of patients in this study were again quite similar to more recently reported MMPI-2 profiles for men

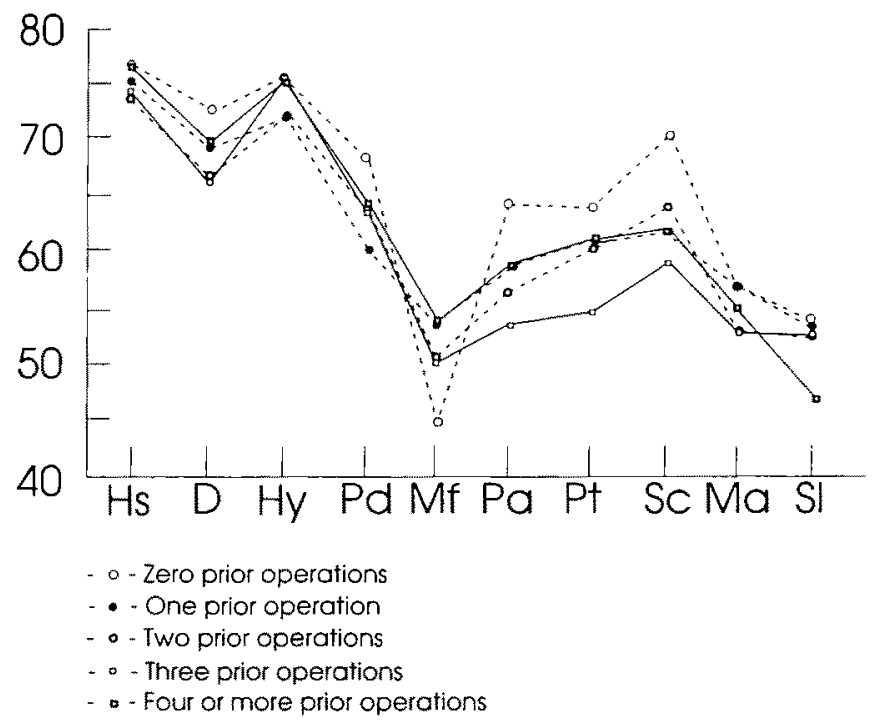

Fig. 1. Number of prior unsuccessful operations for back pain and average response profiles on the MMPI. Validity scales between $T=52$ and $T=62$ for all groups. 
and women with chronic pain (Keller and Butcher, 1991), a finding which is not surprising given the relatively high concordance (97\%) between MMPI and MMPI-2 profiles which have scales $\mathrm{Hs}$ and $\mathrm{Hy}$ with the highest elevations. Thus, it would be expected that this MMPI-2 two-point profile in back pain patients will have clinical ramifications similar to those reported by studies using the original MMPI (Graham, Timbrook, Ben-Porath, \& Butcher, 1991).

For patients with a history of prior interventions for back pain, these results suggest that the somatogenic pain profile continues to be supported as an indicator of decreased prognostic optimism for further surgical intervention. There does not, however, appear to be a differential relationship between MMPI scale measures and the number of previously failed surgeries, thus not indicating any correspondence of increased scores on MMPI scales to greater risk of surgical failure. Thus, no linear relationship between scale elevation and risk status was demonstrated. The presence of the somatogenic profile, in and of itself, appears sufficient to indicate a risk of surgical treatment failure, confirming the findings of Pheasant et al. (1979).

It is not clear, from the present test data, whether the somatogenic profile is significantly associated with surgical failure in patients without a history of prior medical/surgical intervention. In this study, those patients without such a history were selected because medical personnel considered them to have sufficient psychiatric history to warrant further presurgical evaluation. Nevertheless, the similarity between the MMPI profiles for these patients and those for patients with prior surgical exposure suggests a similar risk of poor outcome.

A future approach refining the predictive validity of the MMPI to surgical outcome should involve self-assessment of pain relief and a decrease in movement restriction on the part of those patients undergoing surgical procedures. This appears to be the next logical step in building upon the literature, which generally employs physician ratings of procedural success. We are actively pursuing such a study. At this time, however, the bulk of evidence from the literature, as well as our own results, suggests caution and risk/benefit analysis of contemplated procedures for patients with back pain who produce the MMPI somatogenic pattern of response.

\section{REFERENCES}

Blumetti, A. E., \& Modesti, L. M. (1976). Psychological predictors of success or failure of surgical intervention for intractable back pain. In J. J. Bonica \& D. Albe-Fessard (Eds.), Advances in pain research and therapy, Vol. 1. New York: Raven Press. 
Bradley, L. A., Prokop, C. K., Margolis, R., \& Gentry, W. D. (1978). Multivariate analyses of the MMPI profiles of low back pain patients. Journal of Behavioral Medicine, 1, 253-272.

Butcher, J. N., Dahistrom, W. G., Graham, J. R., Tellegen, A. M., \& Kaemmer, B. (1989). MMPI-2 Manual for Administration and Scoring. Minneapolis: University of Minnesota Press.

Chapman, C. R., \& Bonica, J. J. (1985). Chronic pain. Upjohn.

Graham, J. R., Timbrook, R. E., Ben-Porath, Y. S., \& Butcher, J. N. (1991). Code-type congruence between MMPI and MMPI-2: Separating fact from artifact. Journal of Personality Assessment, 57, 205-215.

Greene, R. L. (191). MMPI-2/MMPI; An interpretive manual. Boston: Allyn and Bacon.

Hathaway, S. R., \& McKinley, J. C. (1983). The Minnesota Multiphasic Personality Inventory Manual. New York: Psychological Corp.

Keller, L. S., \& Butcher, J. N. (1991). Assessment of Chronic Pain Patients with the MMPI-2. Minneapolis: University of Minnesota Press.

Maruta, T., Swanson, D. W., \& Swenson, W. M. (1976a). Low back pain patients in a psychiatric population. Mayo Clinic Proceedings, 51, 57-61.

Maruta, T., Swanson, D. W., \& Swenson, W. M. (1976b). Pain as a psychiatric symptom: Comparison between low back pain and depression. Psychosomatics, XVII, 123-127.

Pheasant, H. C., Gilbert, D., Goldfarb, J., \& Herron, L. (1979). The MMPI as a predictor of outcome in low-back surgery. Spine, 4, 78-84.

Spengler, D., \& Greeman, D. (1979). Patient selection for lumbar discetomy, an objective approach. Spine, $4,129$.

Sternbach, R. A. (1974). Pain patients: Traits and treatment. New York: Academic Press.

Stembach, R. A., Wolf, S. R., Murphy, R. W., \& Akeson, W. H. (1973). Aspects of chronic low back pain. Psychosomatics, 14, 52-56.

Strassberg, D. S., Reimherr, F., Ward, M., Russell, S., \& Cole, A. (1981). The MMPI and chronic pain. Journal of Consulting and Clinical Psychology, 49, 220-226.

Strassberg, D. S., Tilley, D., Bristone, S., \& Oei, T.P.S. (1992). The MMPI and chronic pain: A cross-cultural view. Psychological Assessment, 4, 493-497.

Wifling, F. J., Klonoff, H., \& Kokan, P. (1973). Psychological, demographic and orthopaedic factors associated with prediction of outcome of spinal fusion. Clinical Orthopaedics and Related Research, 90, 153-160.

Wiltse, L. L. (1975). Predicting the success of low back surgery by the use of preoperative psychological tests. Joumal of Bone and Joint Surgery, 57B, 259.

Wiltse, L. L., \& Rocchio, P. D. (1975). Preoperative psychological tests as predictors of success of chemonucleolysis in the treatment of the low-back syndrome. Journal of Bone and Joint Surgery, 57-A, 478-483.

Zachary, R. A. (1991). Shipley Institute of Living Scale. Los Angeles: Western Psychological Services. 